\title{
A REPRESENTATION THEOREM FOR FUNCTIONS HOLOMORPHIC OFF THE REAL AXIS
}

\author{
BY
}

\section{ALBERT BAERNSTEIN II}

\begin{abstract}
Let $f$ be holomorphic in the union of the upper and lower half planes, and let $p \in[1, \infty)$. We prove that there exists an entire function $\varphi$ and a sequence $\left\{f_{n}\right\}$ in $L^{p}(R)$ satisfying $\left\|f_{n}\right\|_{p}^{1 / n} \rightarrow 0$ such that

$$
f(z)=\varphi(z)+\sum_{n=0}^{\infty} \int_{-\infty}^{\infty}(t-z)^{-n-1} f_{n}(t) d t .
$$

This complements an earlier result of the author's on representation of function holomorphic outside a compact subset of the Riemann sphere. A principal tool in both proofs is the Köthe duality between the spaces of functions holomorphic on and off a subset of the sphere. A corollary of the present result is that each hyperfunction of one variable can be represented by a sum of Cauchy integrals over the real axis.
\end{abstract}

1. In [1] we considered functions holomorphic in the (extended) complement of a compact subset $K$ of the complex plane. We proved that, when $K$ is locally connected, each such function $f$ which vanishes at $\infty$ has a representation

$$
f(z)=\sum_{n=0}^{\infty} \int_{K}(t-z)^{-n-1} d \mu_{n}(t) \quad(z \notin K)
$$

where the $\mu_{n}$ are complex Borel measures on $K$ satisfying $\lim _{n \rightarrow \infty}\left\|\mu_{n}\right\|^{1 / n}=0$. In other words, a function holomorphic in a neighborhood of $\infty$ can, under fairly general conditions, be represented by a sum of Cauchy integrals over its singular set.

In this paper we consider functions $f$ holomorphic in $C-R$, where $C$ denotes the finite complex plane and $R$ denotes the real axis. Thus $f$ is in fact a pair of functions $f_{+}$and $f_{-}$, independently prescribed, one holomorphic in the upper half plane, the other in the lower half plane. The singular set is now $R \cup\{\infty\}$. There are very few such $f$ having a representation (1) with $\left\|\mu_{n}\right\|^{1 / n} \rightarrow 0$ and $R$ in place of $K$, since those that do satisfy

$$
|f(z)| \leqq \sum_{n=0}^{\infty}|\operatorname{Im} z|^{-n-1}\left\|\mu_{n}\right\| .
$$

Thus, they are bounded in every region $|\operatorname{Im} z| \geqq \delta>0$ and tend to zero as $|\operatorname{Im} z|$ $\rightarrow \infty$.

Received by the editors May 4, 1971 and, in revised form, July 15, 1971.

AMS 1970 subject classifications. Primary 30A86; Secondary 46E10, 46F15.

Key words and phrases. Integral representation of holomorphic functions, Cauchy integrals, Köthe duality, hyperfunction. 
On the other hand, we are going to prove the following.

THeORem 1. Let $f$ be holomorphic in $C-R$. Then for any $p, 1 \leqq p<\infty$, there exists a sequence $\left\{f_{n}\right\}$ in $L^{p}(R)$ satisfying $\left\|f_{n}\right\|_{p}^{1 / n} \rightarrow 0$ and an entire function $\varphi$ such that

$$
f(z)=\varphi(z)+\sum_{n=0}^{\infty} \int_{-\infty}^{\infty}(t-z)^{-n-1} f_{n}(t) d t \quad(z \in C-R)
$$

Here \|\|$_{p}$ denotes the norm in $L^{p}(R)$. To see that the sum on the right converges, let $q$ be such that $p^{-1}+q^{-1}=1$. It is not hard to show that

$$
\int_{-\infty}^{\infty}|t-z|^{-(n+1) q} d t \leqq|\operatorname{Im} z|^{1-(n+1) q} \int_{-\infty}^{\infty}\left(1+t^{2}\right)^{-q / 2} d t
$$

for $1<q<\infty, n \geqq 0$. So, by Hölder's inequality, we have

$$
\left|\int_{-\infty}^{\infty} f_{n}(t)(t-z)^{-n-1} d t\right| \leqq C\left\|f_{n}\right\|_{p}|\operatorname{Im} z|^{q-1-n-1}
$$

where $C$ depends on $q$ only. When $p=1$, (3) obviously holds, with $C=1$. It follows from (3) that the sum in (2) converges uniformly, and is bounded, on the sets $|\operatorname{Im} z| \geqq \delta>0$.

If we start with a function $f_{+}$holomorphic in the upper half plane $\Pi$, then it follows from Theorem 1 that $f_{+}$has a representation (2) for $z \in \Pi$. Simply apply the theorem to the function $f$ defined to be $f_{+}$in $\Pi$ and (say) zero in the lower half plane.

2. The theorem asserts that a function holomorphic in $C-R$ can be split into two parts, one of which (the sum of the Cauchy integrals) carries all the finite singularities and has a "weak singularity" at $\infty$ (it is bounded on the tangent half planes $|\operatorname{Im} z| \geqq \delta>0$ ). This splitting off leads, via conformal mapping, to the following result for functions in the unit disk.

COROLlaRY 1. Let $f(w)$ be holomorphic in $|w|<1$. Then there exists a function $\psi$, holomorphic on the sphere except at $w=1$, such that $f(w)-\psi(w) \rightarrow 0$ as $w \rightarrow 1$ inside any Stolz angle with vertex at $w=1$, and $f-\psi$ is bounded in each tangent circle $|w-r|<1-r, 0<r<1$.

Proof. Apply Theorem 1 , with $p=1$, to $f^{*}$, where

$$
\begin{aligned}
f^{*}(z) & =f((z+i) /(z-i))) & & (\operatorname{Im} z>0), \\
& =0 & & (\operatorname{Im} z<0) .
\end{aligned}
$$

Let $h$ be the sum of the Cauchy integrals. We have already observed that $h$ is bounded on the tangent half planes $|\operatorname{Im} z| \geqq \delta>0$. Moreover, from (3), with $q=\infty$, we see that $h(z)=O\left(|\operatorname{Im} z|^{-1}\right)$ as $|\operatorname{Im} z| \rightarrow \infty$. The corollary follows. 
M. Sato [4] introduced the class of hyperfunctions. These generalize Schwartz distributions. Loosely speaking, a hyperfunction (of one variable) is supposed to be like a "boundary jump" $f(x+i 0)-f(x-i 0)$ of some function holomorphic in $C-R$. Precisely, the vector space of hyperfunctions is defined to be the quotient of the space of all functions holomorphic in $C-R$ modulo the space of all entire functions. From Theorem 1 we obtain the following result concerning representation of hyperfunctions by integrals over the real axis.

COROLLARY 2. Every hyperfunction on $R$ has a representing holomorphic function of the form

$$
\sum_{n=0}^{\infty} \int_{-\infty}^{\infty} f_{n}(t)(t-z)^{-n-1} d t \quad(\operatorname{Im} z \neq 0)
$$

with $\left\|f_{n}\right\|_{p}^{1 / n} \rightarrow 0$ as $n \rightarrow \infty, 1 \leqq p<\infty$.

3. The main idea in the proof of Theorem 1 is the duality between the spaces of functions holomorphic on and off a subset of the Riemann sphere, which was developed by Köthe [2]. We will also use some facts from the theory of locally convex spaces [5]. In particular, we will use the following criterion for a linear map between Fréchet spaces to be onto.

Proposition. Let $E$ and $F$ be Fréchet spaces and let $T$ be a linear map from $E$ into $F$. If $\left(T^{\prime}\right)^{-1}(B)$ is equicontinuous in $F^{\prime}$ whenever $B$ is an equicontinuous set in $E^{\prime}$, then $T(E)=F$.

Here $E^{\prime}$ and $F^{\prime}$ denote the topological dual spaces and $T^{\prime}$ denotes the adjoint map. Note that "equicontinuous" can be interchanged with "weakly bounded," since Fréchet spaces are barreled.

Proof. According to [6, p. 198], $T$ is onto if (and only if) the closure of $T(U)$ is a zero-neighborhood in $F$ whenever $U$ is a convex circled zero-neighborhood in E. Since

$$
T(U)^{0}=\left(T^{\prime}\right)^{-1}\left(U^{0}\right)
$$

where ${ }^{0}$ denotes the polar with respect to one of the dualities $\left\langle E, E^{\prime}\right\rangle$ or $\left\langle F, F^{\prime}\right\rangle$, and $U^{0}$ is equicontinuous, it follows from the hypothesis that $T(U)^{0}$ is equicontinuous, hence $T(U)^{00}$ is a zero-neighborhood in $F$. For convex sets in $E$ the closure in the original topology coincides with the closure in the weak topology. Since $T(U)^{00}$ is the weak closure of $T(U)$, we are done.

4. Proof of the theorem. We introduce three Fréchet spaces, $E_{1}, E_{2}$, and $F$. $E_{1}$ is the space of all entire functions and $F$ is the space of all functions holomorphic in $C-R$. These two spaces are given the usual topology of uniform convergence on compact subsets (of $C$ and $C-R$, respectively). $E_{2}$ is the space of all sequences $\left\{f_{n}\right\}_{0}^{\infty}$ in $L^{p}(R)$ such that $\left\|f_{n}\right\|_{p}^{1 / n} \rightarrow 0$ as $n \rightarrow \infty$. It is topologized by the sequence of 
norms \|\|$_{k}$ defined by

$$
\left\|\left\{f_{n}\right\}\right\|_{k}=\sup _{n} k^{n}\left\|f_{n}\right\|_{p} \quad(k=1,2, \ldots) .
$$

Then $E_{2}$ is a locally convex metrizable space, and, as the reader may easily check, it is complete. Spaces like $E_{2}$ were studied in [3].

Define the linear map $T: E_{1} \oplus E_{2} \rightarrow F$ by

$$
T\left(f \oplus\left\{f_{n}\right\}\right)(z)=f(z)+\sum_{n=0}^{\infty} \int_{-\infty}^{\infty}(t-z)^{-n-1} f_{n}(t) d t \quad(z \in C-R) .
$$

$T$ is continuous, because if $K$ is a compact subset of $C-R$, then, if

$$
k^{-1} \leqq \frac{1}{2} \inf \{|\operatorname{Im} z|: z \in K\},
$$

we deduce from (3) that

$$
\left\|T\left(f \oplus\left\{f_{n}\right\}\right)\right\|_{K} \leqq\|f\|_{K}+C^{\prime}\left\|\left\{f_{n}\right\}\right\|_{k}
$$

where $C^{\prime}$ depends only on $K$ and $p$, and \|\|$_{K}$ denotes sup over $K$.

The conclusion of Theorem 1 is that $T$ is onto. We want to deduce this from the proposition, so we look for concrete representations of the dual spaces.

First of all, for any pair of locally convex spaces $E_{1}$ and $E_{2}$, we can identify $\left(E_{1} \oplus E_{2}\right)^{\prime}$ and $E_{1}^{\prime} \oplus E_{2}^{\prime}$ by the rule

$$
\left\langle x_{1} \oplus x_{2}, y\right\rangle=\left\langle x_{1}, y_{1}\right\rangle+\left\langle x_{2}, y_{2}\right\rangle
$$

where $x_{i} \in E_{i}, y \in\left(E_{1} \oplus E_{2}\right)^{\prime}$, and $y_{i} \in E_{i}^{\prime}$ is the restriction of $y$ to $E_{i} \oplus\{0\}$.

Next, if $G$ is an open subset of the Riemann sphere $S$ with $\infty \notin G$, and $E$ is the space of all functions holomorphic in $G$, with the topology of uniform convergence on compact subsets, then, by Köthe's theory [2], $E^{\prime}$ can be identified with the spaces of all (equivalence classes of) functions $g$ holomorphic in some (variable) neighborhood of $S-G$ which vanish at $\infty$. The duality is given by

$$
\langle f, g\rangle=\frac{1}{2 \pi i} \int_{\Gamma} f(z) g(z) d z \quad(f \in E)
$$

where $\Gamma$ is the union of finitely many simple closed rectifiable curves in $G \cap V$, $V$ being a neighborhood of $S-G$ in which $g$ is holomorphic, and $\Gamma$ is such that

$$
\begin{aligned}
\int_{\Gamma}(t-z)^{-1} d t & =2 \pi i & & (z \in G-V), \\
& =0 & & (z \in S-G) .
\end{aligned}
$$

In our case, $E_{1}^{\prime}$ can be identified with the space of functions holomorphic in some neighborhood of $\infty$, and $F^{\prime}$ can be identified with the space of functions holomorphic outside some compact subset of $C-R$. In both cases, the functions vanish at $\infty$. Note that $F^{\prime} \subset E_{1}^{\prime}$. 
The dual of $E_{2}$ can be identified with the set of all sequences $\left\{g_{n}\right\}$ in $L^{q}$ such that $\left\|g_{n}\right\|_{q}=O\left(k^{n}\right)(n \rightarrow \infty)$ for some positive integer $k$. The duality is given by

$$
\left\langle\left\{f_{n}\right\},\left\{g_{n}\right\}\right\rangle=\sum_{n=0}^{\infty} \int_{-\infty}^{\infty} f_{n}(t) g_{n}(t) d t .
$$

These statements are easy to prove and are left to the reader. (Or, the proof of Proposition 3 of [3, p. 45] can be adapted.)

Define the linear map $T_{0}: F^{\prime} \rightarrow E_{1}^{\prime} \oplus E_{2}^{\prime}$ by

$$
T_{0} g=g \oplus\left\{(-1)^{n}(n !)^{-1} D^{n} g\right\}_{0}^{\infty},
$$

where $D^{n} g$ denotes the $n$th derivative of $g$, restricted to $R$. To see that the sequence on the right satisfies the growth condition for $E_{2}^{\prime}$, select a pair of simple closed rectifiable curves, one in the upper half plane, the other in the lower half plane, such that $g$ is holomorphic outside a compact subset of the union of the interiors. Orient both curves counterclockwise, and call their union $\Gamma$. Then we have (since $g$ vanishes at $\infty$ )

$$
D^{n} g(t)=-\frac{n !}{2 \pi i} \int_{\Gamma} g(z)(z-t)^{-n-1} d z \quad(t \in R) .
$$

Take $h \in L^{p}$ with $\|h\|_{p} \leqq 1$. Using Fubini's theorem and the estimate used in (3) for the $L^{q}$ norm of $(t-z)^{-n-1}$, we find

$$
\int_{-\infty}^{\infty}|h(t)|\left|D^{n} g(t)\right| d t \leqq C n !(2 \pi)^{-1}\left(\int_{\Gamma}|g(z)||d z|\right) k^{n+1-q^{-1}}
$$

where $C$ is the constant of (3) and $k^{-1} \leqq \inf \{|\operatorname{Im} z|: z \in \Gamma\}$. It follows from this that $\left\{(-1)^{n}(n !)^{-1} D^{n} g\right\} \in E_{2}^{\prime}$.

We claim that $T_{0}$ can be identified with $T^{\prime}$. Take $f \in E_{1},\left\{f_{n}\right\} \in E_{2}$, and $g \in F^{\prime}$. Then, writing \langle\rangle$_{E}$ to denote $\left\langle E, E^{\prime}\right\rangle$ duality, we have

$$
\begin{aligned}
\left\langle f \oplus\left\{f_{n}\right\}, T_{0} g\right\rangle_{E_{1} \oplus E_{2}} & =\langle f, g\rangle_{E_{1}}+\left\langle\left\{f_{n}\right\},\left\{(-1)^{n}(n !)^{-1} D^{n} g\right\}\right\rangle_{E_{2}} \\
& =\frac{1}{2 \pi i} \int_{\Gamma} f(z) g(z) d z+\sum_{n=0}^{\infty} \int_{-\infty}^{\infty}(-1)^{n}(n !)^{-1} D^{n} g(t) f_{n}(t) d t
\end{aligned}
$$

where $\Gamma$, which depends on $g$, is the same as in (5). (This path is a suitable one for effecting the Köthe duality in both $\left\langle E_{1}, E_{1}^{\prime}\right\rangle$ and $\left\langle F, F^{\prime}\right\rangle$.)

On the other hand, writing

$$
F_{n}(z)=\int_{-\infty}^{\infty}(t-z)^{-n-1} f_{n}(t) d t
$$

we have

$$
\begin{aligned}
\left\langle T\left(f \oplus\left\{f_{n}\right\}\right), g\right\rangle_{F} & =\left\langle f+\sum F_{n}, g\right\rangle_{F}=\langle f, g\rangle_{F}+\sum\left\langle F_{n}, g\right\rangle_{F} \\
& =\frac{1}{2 \pi i} \int_{\Gamma} f(z) g(z) d z+\frac{1}{2 \pi i} \sum_{n} \int_{\Gamma} F_{n}(z) g(z) d z
\end{aligned}
$$


From Fubini's theorem and (5) it follows that

$$
\frac{1}{2 \pi i} \int_{\Gamma} F_{n}(z) g(z) d z=(-1)^{n}(n !)^{-1} \int_{-\infty}^{\infty} f_{n}(t) D^{n} g(t) d t,
$$

hence the right-hand sides of (6) and (7) are the same. Thus $T_{0}$ and $T^{\prime}$ can be identified, as asserted.

To finish the proof, it suffices, by the proposition, to show that $T_{0}^{-1}(B)$ is equicontinuous in $F^{\prime}$ whenever $B$ is equicontinuous in $E_{1}^{\prime} \oplus E_{2}^{\prime}$. We can assume $B=B_{1} \oplus B_{2}$, where $B_{1}$ is the set of all functions holomorphic in $|z|>\rho$, continuous and $\leqq 1$ in $|z| \geqq \rho$, and vanishing at $\infty$, for some fixed $\rho$ [2, Satz 12, p. 39], while $B_{2}$ is any superset of the polar of $\left\{\left\{f_{n}\right\} \in E_{2}:\left\|\left\{f_{n}\right\}\right\|_{k} \leqq 1\right\}$ for some fixed positive integer $k$. We choose

$$
B_{2}=\left\{\left\{g_{n}\right\}:\left\|g_{n}\right\|_{q} \leqq k^{n}, n=0,1,2, \ldots\right\} .
$$

A set in $F^{\prime}$ is equicontinuous if and only if it is contained in one of the form

$$
\{h: h \text { is holomorphic in } S-K \text {, }
$$

$$
\text { continuous on the closure of } \left.S-K \text {, and }\|h\|_{S-K} \leqq M\right\}
$$

for some fixed compact subset $K$ of $C-R$ and some fixed constant $M$.

If $g \in F^{\prime}$ then $T_{0} g \in B_{1} \oplus B_{2}$ iff $g \in B_{1}$ and

$$
\left\|D^{n} g\right\|_{q} \leqq n ! k^{n} \quad(n=0,1,2, \ldots) .
$$

From

$$
D^{n} g(t)=-\int_{t}^{2_{\rho}} D^{n+1} g(\tau) d \tau+D^{n} g(2 \rho)
$$

(the $\rho$ used in defining $B_{1}$ ) we deduce by Hölder's inequality and (9) that

$$
\left|D^{n} g(t)\right| \leqq(n+1) ! k^{n+1}(4 \rho)^{1 / p}+\left|D^{n} g(2 \rho)\right|
$$

holds when $-2 \rho<t<2 \rho$. Since $g \in B_{1}$, Cauchy's formula shows that $\left|D^{n} g(2 \rho)\right|$ $\leqq n ! \rho^{-n}$. Thus the series

$$
\sum_{n=0}^{\infty}(n !)^{-1} D^{n} g(t)(z-t)^{n} \quad(-2 \rho<t<2 \rho)
$$

converges whenever $|z-t|<\min \left(k^{-1}, \rho\right)=\sigma$, its sum is independent of $t$, and so provides a single valued analytic continuation of $g$ to the rectangle

$$
Q=\left\{z:|\operatorname{Im} z|<\frac{1}{2} \sigma,|\operatorname{Re} z|<2 \rho\right\} .
$$

Moreover, $|g| \leqq M^{\prime}$ in $Q$, where $M^{\prime}$ depends only on $k, p$, and $\rho$, and $g$ is continuous on the closure of $Q$. Thus we have proved that $T_{0}^{-1}\left(B_{1} \oplus B_{2}\right)$ is contained in the set (8), with $K=\{z:|z| \leqq \rho, z \notin Q\}, M=\max \left(1, M^{\prime}\right)$, and so $T_{0}^{-1}\left(B_{1} \oplus B_{2}\right)$ is equicontinuous. 


\section{REFERENCES}

1. A. Baernstein, Representation of holomorphic functions by boundary integrals, Trans. Amer. Math. Soc. 160 (1971), $27-37$.

2. G. Köthe, Dualität in der Funktionentheorie, J. Reine Angew. Math. 191 (1953), 30-49. MR 15, 132.

3. C. Roumieu, Sur quelques extensions de la notion de distribution, Ann. Sci. École Norm. Sup. (3) 77 (1960), 41-121. MR 22 \#12377.

4. M. Sato, Theory of hyperfunctions. I, J. Fac. Sci. Univ. Tokyo Sect. I 8 (1959), 139-193. MR 22 \#4951.

5. H. Schaefer, Topological vector spaces, Macmillan, New York, 1966. MR 33 \#1689.

6. A. Wilansky, Functional analysis, Blaisdell, New York, 1964. MR 30 \#425.

Department of Mathematics, Syracuse University, Syracuse, New York 13210 